\title{
First-principles calculation of the thermal properties of silver
}

\author{
Jianjun Xie, ${ }^{1}$ Stefano de Gironcoli, ${ }^{2}$ Stefano Baroni, ${ }^{2,3}$ and Matthias Scheffler ${ }^{1}$ \\ ${ }^{1}$ Fritz-Haber-Institut der Max-Planck-Gesellschaft, \\ Faradayweg 4-6, D-14195 Berlin-Dahlem, Germany \\ ${ }^{2}$ SISSA - Scuola Internazionale Superiore di Studi Avanzati and \\ INFM - Istituto Nazionale per la Fisica della Materia, \\ via Beirut 2-4, I-34014 Trieste, Italy \\ ${ }^{3}$ CECAM - Centre Européen de Calcul Atomique et Moléculaire \\ ENS, Aile LR5, 6, Allée d'Italie, 69007 Lyon, France
}

\begin{abstract}
The thermal properties of silver are calculated within the quasi-harmonic approximation, by using phonon dispersions from density-functional perturbation theory, and the pseudopotential plane-wave method. The resulting free energy provides predictions for the temperature dependence of various quantities such as the equilibrium lattice parameter, the bulk modulus, and the heat capacity. Our results for the thermal properties are in good agreement with available experimental data in a wide range of temperatures. As a by-product, we calculate phonon frequency and Grüneisen parameter dispersion curves which are also in good agreement with experiment.
\end{abstract}

65.70.+y, 63.20.-e, 65.50.+m, 65.40.+g

\section{INTRODUCTION}

The study of the temperature dependence of the properties of materials requires a proper account of nuclear motions. Within the framework of density functional theory (DFT) [1], a major breakthrough in this field has been opened by the introduction of ab-initio molecular dynamics by Car and Parrinello [2]. Far from the melting point, however, a more conventional (and yet largely unexplored in practice) approach based on lattice dynamics proves to be both more accurate and computationally efficient. In the harmonic approximation, the crystal free energy is calculated by adding a static contributionwhich is accessible to standard DFT calculations - to a dynamical contribution which is approximated by the free energy of system of harmonic oscillators corresponding to the crystal vibrational modes (phonons). The latter is nowadays conveniently calculated by using densityfunctional perturbation theory (DFPT) [3, A]. In the quasi-harmonic approximation [5 [7], some anharmonic effects can be accounted for by allowing phonon frequencies to depend on crystal volume. Among other advantages, the (quasi-) harmonic approximation allows an explicit account of quantum effects on nuclear motion, which can be important below the Debye temperature. Furthermore, analysis of the normal vibration modes and of their individual contribution to the free energy can explicitly reveal the mechanism driving the thermal expansion, phase transitions, and the crystal stability.
The main concern about the lattice dynamics method is the range of validity of the quasi-harmonic approximation. Calculations based on various semi-empirical models [8 11] as well as on first-principles methods [ 14 12 14] demonstrate that the quasi-harmonic approximation provides a reasonable description of the dynamic properties of many bulk materials below the melting point. Very recently, first-principles calculations on the thermal expansion of some simple $s-p$ metals indicate that the treatment of anharmonic effects at the quasi-harmonic level provides a remarkable good description of the structural and elastic properties of these materials up to their melting points [15].

In this paper we apply the quasi-harmonic approximation to the study of the thermal properties of the $4 d$ noble metal Ag, such as thermal expansion, heat capacity, and temperature dependence of the bulk modulus. To this end, we first calculate the phonon dispersion curves as functions of volume, by using DFPT [3]. Our results demonstrate that all these quantities can be accurately predicted from the present parameter-free method in a wide range of temperatures.

The paper is organized as follows. In Sec. II, we briefly outline our computational framework, as well as some definitions concerning the physical quantities we have investigated. The results of our calculations are then presented and discussed in Sec. III. Finally, in Sec. IV we give our conclusions. 


\section{THEORY}

\section{A. Equation of state and thermal expansion}

For a given temperature, $T$, and volume, $V$, the equilibrium state of an extended system such as a crystal is determined by the condition that the Helmoltz free energy,

$$
F(V, T)=U-T S
$$

is at a minimum with respect to variations of all possible internal degrees of freedom, such as e.g. atomic arrangements and electronic states. Here $U$ and $S$ indicate the internal energy and entropy, respectively. The equation of state of the system is obtained from Eq.(1) by equating the pressure to minus the volume derivative of the free energy:

$$
p=-\left(\frac{\partial F}{\partial V}\right)_{T}
$$

In the quasi-harmonic approximation, $F$ is given by

$$
\begin{aligned}
F(V, T) & =E(V)+F_{\mathrm{vib}}(\omega, T) \\
& \equiv E(V)+k_{\mathrm{B}} T \sum_{\mathbf{q}} \sum_{j} \ln \left\{2 \sinh \left(\frac{\hbar \omega_{j}(\mathbf{q})}{2 k_{\mathrm{B}} T}\right)\right\}
\end{aligned}
$$

where $E$ is the static contribution to the internal energy - which is easily accessible to standard DFT calculations, $F_{v i b}$ represents the vibrational contribution to the free energy, and $\omega_{j}(\mathbf{q})$ is the frequency of the $j$-th phonon mode at wave vector $\mathbf{q}$ in the Brillouin zone (BZ). Anharmonicity is explicitly, though approximately, accounted for by allowing $E(V)$ to deviate from a quadratic behavior and by letting the phonon frequencies depend on volume. Since the temperatures considered here are well below the electronic energy scale, the contribution of the electronic excitations to the thermal expansion is negligible and is not included in the present work. The equation of state (3) can now be written in the form

$$
\begin{aligned}
p(V, T) & =-\frac{\partial E}{\partial V}-\frac{\partial F_{v i b}}{\partial V} \\
& =-\frac{\partial E}{\partial V}+\frac{1}{V} \sum_{\mathbf{q}} \sum_{j} \gamma_{j}(\mathbf{q}) \mathcal{E}\left(\omega_{j}(\mathbf{q})\right)
\end{aligned}
$$

where $\gamma_{j}(\mathbf{q})$ is the Grüneisen parameter corresponding to the $(\mathbf{q}, j)$ phonon mode, defined as:

$$
\gamma_{j}(\mathbf{q})=-\frac{\partial \omega_{j}(\mathbf{q})}{\partial V} \frac{V}{\omega_{j}(\mathbf{q})}
$$

and $\mathcal{E}\left(\omega_{j}(\mathbf{q})\right)$ is the mean vibrational energy of the $(\mathbf{q}, j)$ phonon given by

$$
\mathcal{E}\left(\omega_{j}(\mathbf{q})\right)=\hbar \omega_{j}(\mathbf{q})\left[\frac{1}{2}+\frac{1}{\exp \left(\hbar \omega_{j}(\mathbf{q}) / k_{B} T\right)-1}\right] .
$$

The thermal expansion is obtained directly from the equation of state (4) and the volume thermal expansion coefficient is defined as

$$
\alpha_{V}=\frac{1}{V}\left(\frac{\partial V}{\partial T}\right)_{p}
$$

The temperature dependence of the bulk modulus is obtained from

$$
\begin{aligned}
B(T) & =V\left(\frac{\partial^{2} F}{\partial V^{2}}\right)_{T} \\
& =V \frac{\partial^{2} E}{\partial V^{2}}+V\left(\frac{\partial^{2} F_{v i b}(\omega, T)}{\partial V^{2}}\right)_{T} .
\end{aligned}
$$

Due to anharmonicity, the heat capacity at constant pressure, $C_{p}$, is different from the heat capacity at constant volume, $C_{V}$. The former, which is what experiments determine directly, is proportional to $T$ at high temperature, while the latter goes to a constant which is given by the classical equipartition law: $C_{V} \approx 3 N k_{\mathrm{B}}$, where $N$ is the number of atoms in the system. The relation between $C_{p}$ and $C_{V}$ is [16]

$$
C_{p}-C_{V}=\alpha_{V}^{2}(T) B V T
$$

and $C_{V}$ is given by

$$
C_{V}=k_{B} \sum_{\mathbf{q}} \sum_{j}\left(\frac{\hbar \omega_{j}(\mathbf{q})}{2 k_{B} T}\right)^{2} \frac{1}{\sinh ^{2}\left(\hbar \omega_{j}(\mathbf{q}) / 2 k_{B} T\right)}
$$

\section{B. Computational details}

The static total energy, $E(V)$, and phonon frequencies, $\omega_{j}(\mathbf{q})$, are calculated by using DFT and DFPT respectively, within the local density approximation [17. We use separable norm-conserving pseudopotentials 18,19 together with a plane-wave basis set up to a kineticenergy cutoff of 55 Ry. Sums over occupied electronic 
states are performed by the Gaussian-smearing specialpoint technique 20,21, using $60 \mathbf{k}$ points in the irreducible wedge of $\mathrm{BZ}$. Phonon frequencies are calculated on a (444) regular mesh and Fourier-interpolated in-between. This Fourier interpolation amounts to including real-space inter-atomic force constants up to the nineth shell of neighbors.

\section{RESULTS}

Figure 1 shows the static total energy per atom, $E(V)$, as a function of the lattice constant $a=(4 V)^{\frac{1}{3}}(\mathrm{Ag}$ is a face-centered cubic metal). Our data are fitted to a Murnaghan's equation of state 22]. The resulting lattice constant, $a_{0}=4.05 \AA$, bulk modulus, $B=1.28 \mathrm{Mbar}$, and pressure derivative of the bulk modulus, $\partial B / \partial p=5.66$, agree well with previous theoretical calculations [23. For comparison, the room-temperature experimental data are: $a_{0}=4.08 \AA$ [24] and $B=1.01 \mathrm{Mbar}$ [16].

According to Eq. (4), in order to obtain the equation of state one must first calculate the phonon band structure as a function of volume. In Figure 2 we display the phonon dispersion curves as calculated along several symmetry directions at the minimum of the static energy. Experimental data at room temperature 25] are reported for reference. The effect of temperature on the phonon dispersions will be discussed later in this paper. Figure 3 shows the calculated dispersion curves of the mode Grüneisen parameters of Ag, as defined by Eq. (5), along the same symmetry directions. The dispersions are discontinuous at the $\mathrm{BZ}$ center as a consequence of the anisotropy and polarization dependence of the sound velocities. The Grüneisen parameters of silver are positive throughout the BZ for all branches, thus implying that there is no anomalous negative thermal expansion at low temperature, as in Si [26]. The averaged Grüneisen parameter of silver is 2.6 , in agreement with the experiment value of 2.5 27].

With the static total energy and the Grüneisen parameters in our hands, we can set up the equation of state via Eq. (3). Figure 4 shows the pressure $p(V, T)$ as a function of the lattice parameter $a$ for several temperatures. It can be seen that for a given pressure, the lattice constant increases with the temperature. At room temperature and zero external pressure, the calculated lattice constant is $a_{0}=4.07 \AA$, which is closer to the room temperature experimental value of $4.08 \AA$ [24 than the result of 4.05 $\AA$ derived from the static total energy. As the tempera- ture is increased, a critical temperature $T_{\mathrm{m}}$ exists above which $p(V, T)$ no longer intersects the $p=0$ line and, within the quasiharmonic approximation, the crystal becomes mechanicaly unstable due to the vanishing of the isothermal bulk modulus $-V(\partial p / \partial V)_{T}$ at $T_{\mathrm{m}}$. In Silver this lattice instability occurs at $1370 \mathrm{~K}$, not far from the experimental melting temperature $(1234 \mathrm{~K})$ [16.

The thermal expansion can be derived directly from the equation of state. We have calculated the linear thermal expansion which is defined [28] as

$$
\varepsilon=\frac{\Delta a_{0}}{a_{0}}=\frac{a_{0}(T)-a_{0}(T=293)}{a_{0}(T=293)} .
$$

The results are shown in Figure 5. The agreement between the theoretical and experimental results is very good. Although the calculated equilibrium lattice constant is slightly different from experimental measurement, the temperature dependence of the relative volume changes are described accurately by the present method. The good agreement between theory and experiment holds not only at low temperature, but also near the melting point.

Given the volume as a function of temperature, the temperature dependence of phonon frequencies can be approximately estimated through their volume dependence: $\frac{(\omega(T)-\omega(T=293))}{\omega(T=293)} \approx 3 \gamma \varepsilon$, where $\gamma$ is the Grüneisen parameter (Eq. (5)) and $\varepsilon$ is the linear thermal expansion, (Eq.(11)). In the present case, given the fact that $\varepsilon(T=0) \approx-5 \times 10^{-3}$, and that Grüneisen parameters are tipically $\gamma \approx 2 \sim 3$, phonon frequencies at room temperature are lower than their $T=0$ value by $3 \sim 5 \%$. Figure 6 shows the theoretical phonon dispersions at room temperature (full lines) together with the dispersions, which are already shown in Figure 2 and calculated at the static equilibrium (dashed lines in Figure 6). The comparison with experimental data at room temperature (open circles) 25] is clearly improved and now rather satisfactory.

Figure 7 shows the temperature dependence of the bulk modulus as calculated from Eq. (8). $B_{0}$ denotes the bulk modulus obtained from the static total energy by neglecting the lattice vibrations. At room temperature, we obtain a bulk modulus $B=1.16$ Mbar. Comparing this value with the result derived from static LDA calculations $\left(B_{0}=1.28 \mathrm{Mbar}\right)$, one sees that the agreement with the experimental result $(B=1.01 \mathrm{Mbar})$ [16] is significantly improved.

The calculated heat capacity $C_{p}$ and $C_{V}$ are shown in 
Figure 8. It can be seen that below the Debye temperature $\left(\theta_{D}=215 \mathrm{~K}\right.$ for silver [27]) the difference between $C_{p}$ and $C_{V}$ is very small, while at high temperature, the heat capacity at constant volume $C_{V}$ approaches to the classical value $2.49 \mathrm{Jmol}^{-1} \mathrm{~K}^{-1}$, while the heat capacity at the constant pressure increases monotonously with the temperature. The available experimental data [29] for $C_{p}$ are shown as circles. The agreement between theory and experiment is remarkable in a wide range of temperatures also in this case.

\section{CONCLUSIONS}

In the present paper, we have calculated the thermal properties of silver, such as thermal expansion coefficient, Grüneisen parameters, bulk modulus, and heat capacity, using the the quasi-harmonic approximation within density-functional theory. The equilibrium lattice constant is obtained from the equation of state constructed by the free energy. The volume dependence of frequencies is calculated from the density-functional perturbation theory. The obtained results for the investigated thermodynamic quantities are in good agreement with the available experiment measurements. The calculation suggests that the anharmonic properties of silver can be accurately calculated from this first-principles approach in a wide range of temperature. The application of the present method to the study of other properties of materials such as high pressure effect, crystal stability and phase transitions, is straightforward.

\section{ACKNOWLEDGMENTS}

One of the authors (J.J. Xie) would like to acknowledge the financial support from Alexander von Humboldt foundation in Germany. Two of us (SB and SdG) have done this work in part within the Iniziativa Trasversale Calcolo Parallelo of INFM.
[1] For a review of Density Functional Theory, see e.g.: S. Lundqvist and N.H. March, Eds., Theory of the Inhomogeneous Electron Gas (Plenum, New York, 1978)

[2] R. Car and M. Parrinello, Phys. Rev. Lett. 55, 2471 (1985).

[3] S. Baroni, P. Giannozzi, and A. Testa, Phys. Rev. Lett. 58, 1861 (1987); P. Giannozzi, S. de Gironcoli, P. Pavone, and S. Baroni, Phys. Rev. B 43, 7231 (1991).

[4] A. Fleszar and X. Gonze, Phys. Rev. Lett. 64, 2961 (1990).

[5] R.E. Allen and F. W. de Wette, Phys. Rev. 179, 873 (1969).

[6] A.A. Maradudin, E.W. Montroll, G.H. Weiss, and I.P. Ipatpva, Theory of Lattice Dynamics in the Harmonic Approximation, 2nd ed. (Academic, New York, 1971).

[7] P. Brüesch, Phonons: Theory and Experiments I. (Springer-Verlag, Berlin Heidelberg New York, 1982).

[8] S.M. Foiles and J. B. Adams, Phys. Rev. B 40, 599 (1989).

[9] J.D. Althoff, P.B. Allen, R.M. Wentzcovitch, and J.A. Moriarty, Phys. Rev. B 48, 13253 (1993).

[10] C.H. Xu, C.Z. Wang, C.T. Chan, and K.M. Ho, Phys. Rev. B 43, 5024 (1991).

[11] G.D. Barrera, M.B. Taylor, N.L. Allan, T.H.K. Barron, L.N. Kantorovich, and W.C. Mackrodt, J. Chem. Phys. 107, 4337 (1997).

[12] S. Biernacki and M. Scheffler, Phys. Rev. Lett. 63, 290 (1989).

[13] P. Pavone, K. Karch, O. Schütt, W. Windl, and D. Strauch, P. Giannozzi and S. Baroni, Phys. Rev. B 48, 3156 (1993); see also P. Pavone Lattice Dynamics of Semiconductors from Density-Functional Perturbation Theory (SISSA PhD Thesis, Trieste, 1991), available on the WWW at URL: http://www.sissa.it/cm/PHD.html

[14] P. Pavone, S. Baroni, and S. de Gironcoli, Phys. Rev. B, in press.

[15] A.A. Quong, A.Y. Liu, Phys. Rev. B 56, 7667 (1997).

[16] C. Kittel, Introduction to Solid State Physics, 6th ed., (John Wiley and Sons, New York, 1986).

[17] D.M. Ceperley and B.J. Alder,Phys. Rev. Lett. 45, 566(1980) as parameterized by J.P. Perdew and A. Zunger, Phys. Rev. B 23, 5048 (1981).

[18] L. Kleinman and D.M. Bylander, Phys. Rev. Lett. 48, 1425 (1982).

[19] X. Gonze, R. Stampf, and M. Scheffler, Phys. Rev. B 43, 8503 (1995). 
[20] M. Methfessel and A.T. Paxton, Phys. Rev. B 40, 3616 (1989).

[21] H.J. Monkhorst and J.D. Pack, Phys. Rev. B 13, 5188 (1976).

[22] F.D. Murnaghan, Proc. Natl. Acad. Sci. USA 50, 697 (1994).

[23] S. Narasimhan and M. Scheffler, Z. Phys. Chem. 202, 253 (1997).

[24] Landolt-Börnstein Numerical Data and Functional Relationships in Science and Technology Vol. 13a, 7, (Springer-Verlag Berlin, Heidelberg, New York 1981).

[25] W. Drexel, Z. Phys. 255, 281 (1972).

[26] H. Ibach, Phys. Status Solidi 31, 625 (1969).

[27] K.A. Gschneider, Jr., in Solid State Physics, edited by F. Seitz and D. Turnball (Academic, New York, 1964), Vol. 16, p. 275.

[28] American Institute of Physics Handbook, 3rd ed., 4-130, (McGraw-Hill, New York, 1982).

[29] American Institute of Physics Handbook, 2nd ed., 4-49, (McGraw-Hill, New York, 1963).

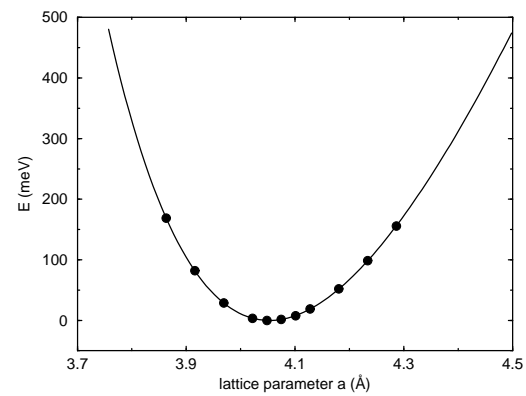

Figure 1, Xie et al

FIG. 1. Static total energy per atom as a function of lattice parameter $a$ 


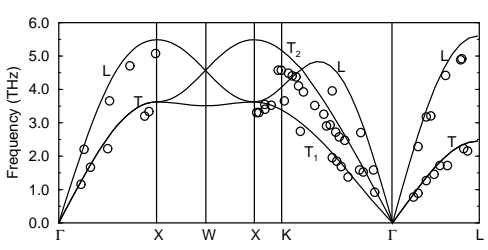

Figan 2 Xiv tat

FIG. 2. Calculated phonon dispersion curves at the lattice parameter corresponding to static equilibrium. Experimental neutron-scattering data 25 are denoted by circles. $\mathrm{T}$ and $\mathrm{L}$ represent transverse modes and longitudinal modes respectively.

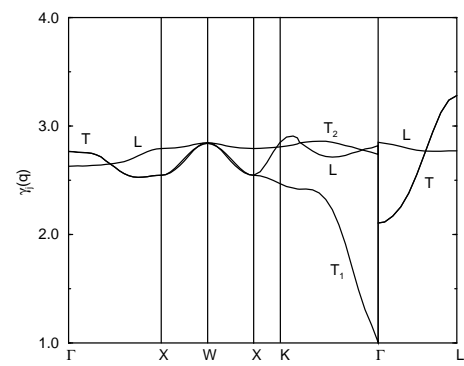

Figure 3. Xie et al

FIG. 3. Calculated dispersion curves of the mode Grüeisnen parameter $\gamma_{j}(\mathbf{q})$ of silver along some symmetry lines in BZ. T and $\mathrm{L}$ denotes the transverse modes and longitudinal modes respectively. 


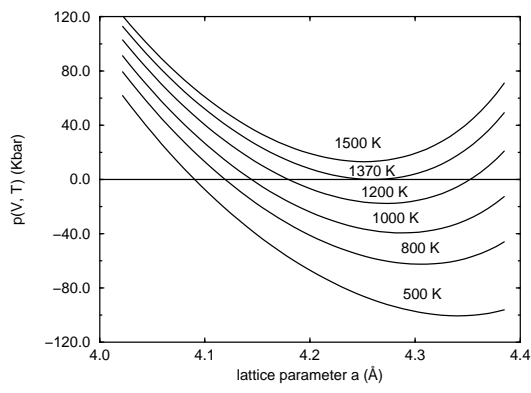

Figure \&, Xie et al

FIG. 4. Applied pressure $P(V, T)$ for silver as a function of lattice parameter $a$ (here $V=a^{3} / 4$ ) for different temperatures.

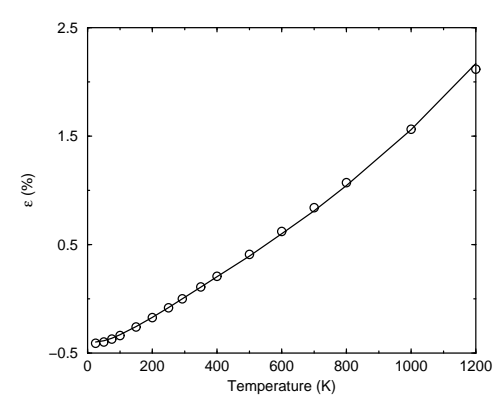

Figure 5, Xie et

FIG. 5. Temperature dependence of the linear thermal expansion for Ag. Solid curve is the calculated result and the circles represent experimental data from Ref. 28]. 


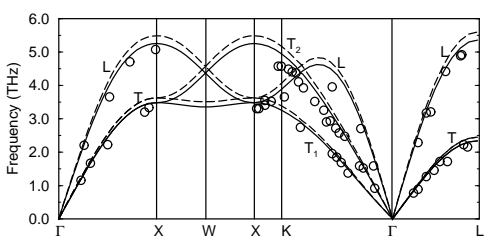

Figan , . Xive tat

FIG. 6. Solid lines: calculated phonon dispersion curves of silver at $T=293 \mathbf{K}$. Dashed lines: calculated phonon dispersions at the lattice parameter corresponding to static equilibrium. Experimental neutron-scattering data 25] are denoted by circles. $\mathrm{T}$ and $\mathrm{L}$ represent transverse modes and longitudinal modes respectively.

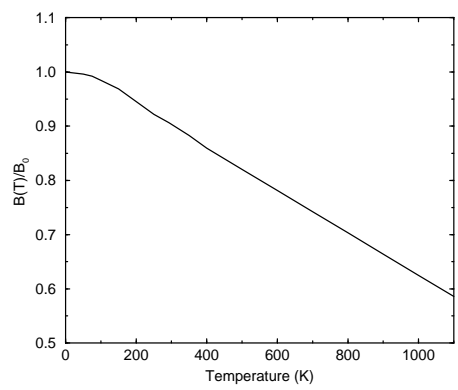

Figure 7 , Xie $e$ al

FIG. 7. Calculated temperature dependence of the ratio of bulk modulus $B(T)$ to $B_{0}$ (obtained from static total energy) for silver. 


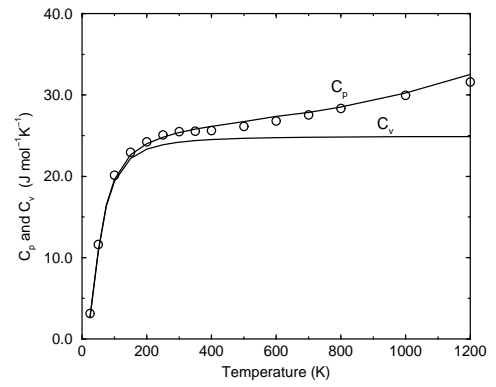

FIG. 8. Calculated temperature dependence of heat capacity of $\mathrm{Ag}$ at constant pressure $\left(C_{p}\right)$ and at constant volume $\left(C_{V}\right)$. The experimental data for $C_{p}$ [29] are denoted by circles. 